\title{
Retour sur le classement des genres à enseigner
}

État des lieux et tentative de clarification

\section{Ecaterina Bulea}

\section{(2) OpenEdition Journals}

Édition électronique

URL : http://journals.openedition.org/pratiques/3843

DOI : $10.4000 /$ pratiques.3843

ISSN : 2425-2042

Éditeur

Centre de recherche sur les médiations (CREM)

\section{Édition imprimée}

Date de publication : 1 juin 2013

Pagination : 201-215

Référence électronique

Ecaterina Bulea, «Retour sur le classement des genres à enseigner », Pratiques [En ligne], 157-158 | 2013, mis en ligne le 18 décembre 2017, consulté le 19 avril 2019. URL : http:// journals.openedition.org/pratiques/3843; DOI : 10.4000/pratiques.3843 


\title{
Retour sur le classement des genres à enseigner. État des lieux et tentative de clarification
}

\author{
Ecaterina Bulea
}

FPSE, Université de Genève

Le problème du classement des genres de textes à enseigner s'est posé dans le champ didactique depuis la mise en œuvre, dans le courant des années 1980, d'une démarche de rénovation préconisant notamment la diversification des sortes de textes à introduire dans les programmes scolaires, au titre de formes d'expression, écrite ou orale, devant progressivement être maîtrisées. Comme en attestent en particulier les articles publiés dans Langue française 74 (1987), Pratiques 62 (1989) et Etudes de linguistique appliquée 83 (1991), de multiples travaux ont alors été réalisés, visant à solliciter les typologies de textes/discours existant dans le champ des sciences du langage, voire à en élaborer de nouvelles versions, et à établir, par adaptation/transposition de ces typologies, un ré-étiquetage et un classement des genres textuels à enseigner. Au cours des années 1990, de nombreux produits de ces travaux ont été implantés dans les systèmes didactiques, en même temps qu'étaient créés et diffusés de nouveaux dispositifs méthodologiques destinés à l'enseignement/apprentissage des conditions d'usage et des régularités d'organisation desdits genres. Mais dès les années 2000, certaines décisions prises par les autorités politico-éducatives de la francophonie ont donné lieu à des replis, partiels et désordonnés, eu égard aux ambitions rénovatrices antérieures; ce qui a généré une considérable confusion quant aux objectifs, aux programmes et aux méthodes de l'ensemble des domaines de l'enseignement du français.

Dans la présente contribution, nous proposerons d'abord une analyse rétrospective de certains aspects de la mise en œuvre initiale de la démarche de diversification des genres textuels : en rappelant la teneur des demandes sociales à l'origine cette démarche, en reformulant les principaux problèmes que posaient - et que posent encore - la confection des typologies et/ou classements théoriques des textes, et en relevant les tendances majeures de leurs adaptations didactiques. Nous fournirons ensuite un exemple concret de la situation actuelle en ce domaine pour ce qui concerne la Suisse romande, en analysant les orientations et les propositions formulées, d'une part dans le document officiel de cadrage général de l'enseignement du français actuellement en vigueur (Plan d'Etudes Romand), d'autre 
part dans l'un des manuels en usage, obligatoire ou conseillé, dans les cantons romands. Sur la base de ces deux types d'analyse, nous formulerons pour clore quelques propositions de clarification théoriques, terminologiques et didactiques.

\section{Besoins de diversification et... de classement}

Les évolutions politico-didactiques récentes semblent témoigner d'un oubli, voire d'un abandon, des deux objectifs qui sous-tendaient le principe de diversification prôné par la rénovation. Le premier objectif, qui paraît quasiment étrange en ce XXI ${ }^{\mathrm{e}}$, avait été formulé avec une particulière clarté par Moreau et Mouvet (1990) : en dépit de la diversité de leur situation socioculturelle, tous les élèves sont aptes à réaliser la plupart des actions langagières requises de la vie en société, mais certains le font sous des formes passablement éloignées des normes explicites ou implicites du français écrit. Dès lors, ce que requiert d'abord la démarche de diversification, c'est que l'on tienne compte de ces capacités initiales des élèves, pour conduire ces derniers à la maîtrise des versions normées de réalisation des actions langagières. Moins directement articulé à ces principes de démocratisation et d'égalité des chances, le second objectif avait trait à la représentativité des genres enseignés à l'école : les programmes scolaires traditionnels ne promouvaient que quelques genres censés faciliter l'accès, soit à la littérature (rédaction et portrait), soit aux œuvres philosophiques (dissertation), et le principe de diversification impliquait en ce domaine de substituer à cette fonction propédeutique une fonction de socialisation : préparer les élèves à la maîtrise des genres qu'ils auraient à utiliser dans leur vie scolaire, puis professionnelle.

Si l'objectif de représentativité était en soi d'une indiscutable pertinence, sa mise en œuvre en situation scolaire était d'une tout aussi indiscutable complexité : dès lors que les genres constituent des ensembles flous, que l'on ne peut tous les enseigner, que certains sont trop complexes et d'autres trop prosaïques, que d'autres encore entretiennent entre eux d'étroites relations familiales, etc., comment sélectionner, classer et dénommer les genres à enseigner ? La réponse à une telle question impliquait tout naturellement que l'on prenne connaissance de l'état des réflexions conduites en ce domaine dans le champ des sciences du langage et/ou de la littérature.

\subsection{Malaise(s) dans la classification}

Dans un important article portant le titre ci-dessus (sans marque du pluriel), Chiss (1987) avait tout d'abord relevé un ensemble de positions émanant de théoriciens de la littérature selon lesquelles les démarches de classement et de généralisation que le structuralisme linguistique avait introduites comme gages de scientificité n'étaient guère applicables au domaine du texte, et ne l'étaient nullement s'agissant des textes proprement littéraires. Le premier de leurs arguments était que tout texte est radicalement unique, même s'il est traversé de stéréotypes et porteur de valeurs socioculturelles ; unicité qui, selon Barthes (1984) par exemple, rendait vaine ou illusoire toute science générale du texte. Le second de leurs arguments avait trait à la multiplicité et à l'hétérogénéité des critères pouvant légitimement être pris en considération pour constituer les catégories de genres textuels; variabilité débouchant immanquablement sur une multiplicité de typologies incontrôlables et impraticables, comme le soutenait notamment Maingueneau : 
Pour maîtriser un tant soit peu 1'univers discursif on utilise constamment des typologies fonctionnelles (discours juridique, religieux, politique...) et formelles (discours narratif, didactique...) qui s'avèrent aussi inévitables que dérisoires. [...] On est condamné à penser un mélange inextricable de même et d'autre, un réseau de rapports constamment ouvert. Rien d'étonnant si les typologies, dès qu'on les scrute d'un peu près et qu'on veut les appliquer, volent en éclats, laissant apparaître un immense entrelacs de textes dans lesquels seules les grilles idéologiques d'une époque, d'un lieu donnés, ou les hypothèses qui fondent une recherche, peuvent introduire un ordre. (Maingueneau, 1984, p. 16)

L'émergence et l'expansion de la grammaire générative ont suscité l'apparition d'un courant d'orientation radicalement contraire au précédent, visant à l'identification de l'ensemble des règles d'organisation/génération des textes. Illustrées en particulier par les travaux de Van Dijk ( $c f .1972$ ), ces «grammaires de textes » cherchaient à rendre compte des capacités présumées des sujets à différencier les suites de phrases formant un texte cohérent de celles conduisant à un texte incohérent, mais aussi de leur capacité à reconnaître des parentés structurales entre des textes superficiellement différents. Si les approches de ce type ont pu être exploitées par certains courants de psycholinguistique en raison de leur orientation cognitiviste ( $c f$. Kintsch \& Van Dijk, 1978), elles n'ont, pour cette même raison de centration sur des compétences générales, fourni aucun véritable apport à la question du statut et du traitement possible des différences constitutives de la généricité textuelle. Cette question a par contre été abordée de front, au cours des années 1970 et 1980, par un ensemble de linguistes germanophones adhérant à une conception du langage radicalement opposée à celle de Chomsky en ce qu'elle posait la primauté de l'activité discursive eu égard au système générateur de phrases, et qu'elle se centrait sur les relations d'interdépendance entre l'organisation textuelle d'une part, les contextes communicatifs et les activités humaines concernées d'autre part. Et les approches de ce type ont tout naturellement donné lieu à des typologies de textes (que l'on qualifierait plutôt aujourd'hui de « classements des genres ») fondées sur le critère fonctionnel du mode d'interaction avec leur contexte situationnel et praxéologique ( $c f$. Schmidt, 1976; Werlich, 1975).

La redécouverte de l'œuvre de Volochinov, au travers - et en dépit - de l'indécente promotion du bakhtinisme ${ }^{(1)}$, a engendré un autre courant de recherche adhérant à la thèse, formulée par Volochinov dans Le discours dans la vie et le discours dans la poésie (1926/1981), de la communauté/continuité entre textes profanes et textes littéraires, et articulant, en écho au programme méthodologique présenté dans Marxisme et philosophie du langage (1929/2010, p. 321-322), des niveaux d'analyse descendants : traiter d'abord des sphères d'activité humaines dans lesquelles s'insèrent les interactions verbales; identifier ensuite les dimensions génériques des textes effectivement produits dans ces interactions ; examiner enfin l'organisation interne de chaque texte, quel que soit le genre dont il relève. Illustré en particulier par les démarches de la linguistique textuelle ( $c f$. Adam, 1990 et 2011) et de l'interactionnisme socio-discursif ( $c f$. Bronckart, 1997 ; Bronckart et al., 1985), ce courant a repris une part importante du projet de

(1) Comme l'ont montré Bronckart \& Bota (2011), les thèmes essentiels du bakhtinisme (généricité étendue, dialogisme, attitude responsive active, polyphonie, etc.) ont été conçus et développés dans les travaux réalisés par Volochinov dans les années 1920, et « reformulés » bien plus tard dans certains textes signés de Bakhtine, en même temps que ce dernier tentait de s'attribuer la paternité des œuvres de Volochinov et de Medvedev. 
l'analyse de discours «à la française » ( $c f$. Todorov, 1978) et intégré également les critères de classement des textes qui avaient été élaborés par Benveniste (1966), Weinrich (1973) ou Simonin-Grumbach (1975). Au-delà de leurs particularités, les travaux conduits dans cette perspective ont en commun de souligner la primauté sémiotique de la généricité, et de poser l'existence de deux plans de différenciation des textes. Le premier plan (global) est celui du genre dont relève un texte, qui est saisi dans la relation de ce texte avec les paramètres externes que constituent les types d'activités humaines impliquées, les contextes de communication ou encore les visées de l'auteur. Le second plan (local) est celui des formes d'organisation attestables au sein d'un genre et descriptibles sur la base de leurs propriétés linguistiques internes, formes qui sont saisies, dans le premier cadre théorique sous l'angle des modes de structuration du contenu thématique (narration, description, argumentation, explication, etc.), dans le second cadre sous l'angle du mode de positionnement énonciatif exhibé ${ }^{(2)}$ (discours interactif, discours théorique, récit interactif, narration).

Les deux derniers courants évoqués ci-dessus (germanophones et francophones) ont produit des classements et/ou typologies de textes, qui se sont ajoutés à — ou sont entrés en concurrence avec — d'autres propositions à même visée que nous ne pourrons évoquer ici. Ce foisonnement a alors quasi naturellement généré des travaux méta-classificatoires : dans la démarche séminale en ce domaine, Schneuwly (1988) distinguait six catégories, mais un accord semble ensuite s'être établi ( $c f$. Petitjean, 1989; Schneuwly, 1991) sur l'existence de trois grands procédés classificatoires, fondés respectivement sur des critères d'ordre cognitif ou procédural (mode d'organisation du contenu), d'ordre énonciatif (rapport au contexte communicatif) ou d'ordre fonctionnel (intentions/visées du texte). Et tous les auteurs de ces «typologies de typologies » reconnaissaient, au moins implicitement, la pertinence intrinsèque de chacune de ces orientations classificatoires.

\subsection{Des classements de textes pour l'enseignement}

Dans le cadre de la démarche de diversification, de nombreux formateurs et/ou enseignants ont conçu, testé et mis en œuvre une activité nouvelle de tri de textes dont le but était de sensibiliser les élèves à la diversité des genres, et plus particulièrement de leur faire comprendre que les textes ne diffèrent pas seulement par leur thématique, mais aussi par leurs propriétés linguistiques et compositionnelles. Cette activité se concrétisait notamment en démarches d'identification et de conceptualisation des plus saillantes de ces propriétés différentielles, démarches censées favoriser des apprentissages cognitifs plus généraux, comme le soulignait Garcia-Debanc ( « La pratique du tri de textes développe des apprentissages méthodologiques transversaux, tels que les capacités à observer, comparer, analyser, dégager des indices pertinents pour la différenciation »-1989, p. 6) et comme l'avaient démontré certaines recherches expérimentales (cf. Brassart et al., 1986).

La plupart des promoteurs de ces démarches de tri ont, en dépit de différences d'orientation pédagogique parfois importantes, prôné une même attitude de détachement ou de relativisme eu égard aux propositions théoriques évoquées sous

(2) Ce sont précisément ces modes de positionnement énonciatif qui sont désignés, dans le cadre de l'interactionnisme socio-discursif, par l'expression types de discours, alors que dans le cadre de la linguistique textuelle les modes de structuration du contenu sont désignés par le terme de séquences. 
1.1. Pour Privat \& Vinson par exemple, la multiplicité des typologies théoriques constituait même une sorte d'atout didactique :

Les lacunes, insuffisances ou incertitudes théoriques constituent, en l'état actuel des choses, une situation paradoxalement intéressante : elle place la classe en position véritable de recherche expérimentale (production d'hypothèses génériques, vérifications, modifications, généralisations, etc.) en fonction d'acquis et de projets qui lui sont propres. (1988, p. 4)

Selon Coste, dans le cadre de l'enseignement du FLE les démarches de ce type devaient conduire à sensibiliser les apprenants, simultanément ou de manière coordonnée :- à la centralité du discursif, en tant que rapport que les textes entretiennent avec leur milieu praxéologique et culturel; - à la diversité des textes impliqués dans ces rapports ; - à 1'unicité des phénomènes proprement grammaticaux. Et s'agissant de l'approche de la diversité textuelle, il posait que :

[...] pédagogiquement [...] les classements de textes, inéluctables, seront d'autant moins préjudiciables qu'ils seront multiples, se relativisant les uns les autres, évitant qu'une typologie ne se durcisse et devienne normative. (1991, p. 87)

Schneuwly enfin, soulignant la nécessité des adaptations didactiques, attirait l'attention sur le fait que les typologies textuelles n'étaient pas en elles-mêmes des objets d'enseignement et recommandait une sorte d'allègement ou de banalisation des démarches de tri et de conceptualisation des classes de textes :

Les typologies de textes telles qu'elles existent actuellement ne doivent pas devenir telles quelles des objets de connaissance pour les élèves. [...] Ceci ne signifie pas abandonner, en classe, des observations de différences entre genres ou sortes de textes, par exemple au travers des exercices de tri de textes, mais de telles activités devraient faire référence aux connaissances et au vocabulaire quotidien amplement suffisant dans ce contexte. (1991, p. 134)

La prise de position de Schneuwly ci-dessus évoquée s'inscrit dans le projet d'exploitation de la typologie élaborée par l'unité genevoise de didactique des langues au début des années 1980 et formalisée dans Bronckart et al. (1985). Dans une première phase de ses travaux, cette unité avait élaboré une " grille d'analyse » des propriétés linguistiques de chaque type de discours (voir supra et note 2 pour cette notion), qui était directement destinée à la formation des enseignants ( $c f$. Bain et al., 1982) : l'objectif était que les enseignants prennent conscience de ces spécificités, pour pouvoir sélectionner les textes ou les segments de textes contenant les sous-ensembles d'unités ou de régularités qui constituaient leurs objets d'enseignement. Dans une phase ultérieure, cette unité de recherche a conçu et produit une série de séquences didactiques ayant trait à l'expression écrite ou orale ( $c f$. Dolz \& Schneuwly 1998 ; Schneuwly \& Dolz, 1997), et une part des d'activités incluses dans ces séquences visait à mettre en évidence les relations d'interdépendance entre propriétés des situations de communication et propriétés linguistiques des textes produits. Comme l'indiquait par exemple Bain,

[...] une classification des types ou genres de textes n'a d'intérêt pour le pédagogue que si elle met en relation les types de discours, marqués par l'utilisation de certaines unités linguistiques, et les situations de communication qui vont susciter ou déterminer leur production. [...] Dans ce cas, la typologie risque moins d'être utilisée comme un simple moyen d'étiqueter des textes, activité relativement stérile sur le plan didactique ; elle se présente comme un modèle de fonctionnement 
du discours, à partir duquel 1'enseignant pourra élaborer ses stratégies didactiques. (1987, p. 49)

Dans une perspective explicitement inspirée des travaux de cette unité, Filliolet (1987) a pour sa part proposé un schéma de progression pédagogique, selon lequel les enseignants aborderaient d'abord les types de discours homogènes ou archétypiques, susceptibles de fournir aux élèves des régularités et principes textuels généraux, avant de passer aux textes exhibant des formes diverses de mixité ou d'hétérogénéité.

S'ils ont sans nul doute engendré un enrichissement et une clarification des activités didactiques relatives aux textes, ces démarches ont cependant été obérées par l'imprécision subsistant quant aux rapports entre «genres de textes » et «types de discours », comme en atteste notamment le début de la citation de Bain, supra: les facteurs externes, dont ceux concernant la situation de communication, contribuent certes au choix d'un genre, mais un même genre comporte généralement plusieurs types de discours différents, et des genres textuels distincts peuvent mobiliser les mêmes types de discours. Il n'y a donc pas de lien direct entre les paramètres externes de la communication et les propriétés linguistiques d'un type de discours; et la sous-estimation de ce phénomène nous paraît avoir été la source de multiples difficultés et confusions didactiques, nous y reviendrons sous 3 .

On relèvera encore que dans la plupart des publications traitant d'activités d'analyse ou de classement de textes, les étiquettes de genre appliquées aux textes singuliers étaient celles du vocabulaire de sens commun, et ne présentaient donc aucune forme de rationalité ou de généralité, comme en attestent les articles de Etudes de linguistique appliquée 83 déjà mentionné ; les démarches argumentées de conceptualisation ne portaient que sur la constitution de familles de genres. Mais en ce dernier domaine, à l'exception notable de la classification de Mas \& Turco (1991), les propositions mélangeaient généralement les critères relatifs aux fonctions interactives « pré-textuelles » (faire agir, prescrire, amuser, etc.) et ceux concernant les modes d'organisation de la textualité effectivement produite (narration, description, dialogue, etc.).

\section{2. État des lieux : un carottage en terrain romand}

Au cours de la dernière décennie, la Suisse romande a décidé d'élaborer des programmes scolaires communs aux différents cantons, ce qui s'est concrétisé par l'élaboration d'un Plan d'Etudes Romand (ci-après PER) dont la version définitive a été adoptée par la Conférence Intercantonale de l'Instruction Publique (CIIP) en mai 2010, et qui est progressivement mis en œuvre dans l'ensemble des cantons, avec une échéance de généralisation fixée à l'année scolaire 2014-2015. Ce nouveau programme concerne les trois cycles de la scolarité obligatoire (cycle 1 : élèves de 4 à 8 ans; cycle 2 : élèves de 8 à 12 ans; cycle 3 : élèves de 12 à 15 ans). Pour le cycle 2, il distingue cinq domaines, dont 1'un, intitulé Langues, comporte huit thématiques d'activité. L'étude que nous présenterons ci-dessous porte sur le domaine Langues - Français du cycle 2, et plus particulièrement sur les quatre thématiques compréhension de l'écrit, production de l'écrit, compréhension de l'oral, production de l'oral. Nous analyserons la terminologie utilisée pour désigner les genres textuels, les classements qui en sont proposés, ainsi que les objectifs et la teneur des activités didactiques conseillées. 
Dans le prolongement de ce processus de réorganisation des programmes, les cantons romands ont adopté de nouveaux manuels, qui devaient en principe être compatibles avec les démarches et les contenus d'enseignement préconisés par le PER, mais dont le degré d'obligativité pouvait varier selon les cantons. Pour ce qui concerne le canton de Genève, s'agissant du domaine Langues _ Français, la série Mon manuel de français (ci-après MMF) a été introduite dès la rentrée 2011 comme moyen d'enseignement officiel du cycle 2. Nous examinerons le Livre du maître destiné à ce cycle, plus précisément la partie théorique initiale de celui-ci, commune aux manuels relatifs aux quatre degrés de ce cycle ${ }^{(3)}$. Comme pour le PER, nous y analyserons la terminologie relative aux genres, les classements proposés et les activités décrites.

\subsection{Les genres textuels et leur classement dans le PER}

Pour les quatre thématiques retenues, le PER énonce d'abord les apprentissages communs à tous les genres de textes, qui pourraient être réalisés en exploitant des supports variés (encyclopédie, fiche documentaire, dictionnaire, carte postale, lettre, affiche, journal, livre, etc.). Pour les deux thématiques de compréhension (PER, p. 22 et 42), ces apprentissages consistent en l'identification :- des différents genres textuels ; - des éléments de la situation de communication;- de l'organisation générale du texte ; - de certains aspects du thème ou sujet traité. Pour les deux thématiques de production ( $\mathrm{ibid}$., p. 34 et 48), les apprentissages consistent notamment en :- l'identification de la situation de communication ; - le respect du genre textuel demandé ; - l'organisation du texte selon un modèle donné.

Pour les quatre thématiques, la présentation des objectifs et méthodes des activités à conduire est organisée en fonction d'un "regroupement de genres » qui est introduit sans le moindre commentaire justificatif et qui comporte les six rubriques suivantes : "le texte qui raconte », "le texte qui relate", "le texte qui argumente ", "le texte qui transmet des savoirs", "le texte qui règle des comportements » et «le texte qui joue avec la langue ». La présentation de chacune des rubriques comporte une liste de genres conseillés qui y sont inclus, suivie de l'énoncé de divers apprentissages à réaliser, avec leurs « attentes fondamentales » et des « indications pédagogiques ».

La distinction entre « texte qui raconte » et « texte qui relate » semble tenir au degré de réalité des événements verbalisés, la première classe comportant les genres de fiction ou autorisant la fiction comme le conte merveilleux, le récit d'aventure, la fable, la légende, etc., la seconde comportant les genres organisant des contenus plus « objectifs », comme le récit de vie, le témoignage d'une expérience vécue, le fait divers, la biographie, etc. S'agissant des apprentissages à réaliser, si dans les deux cas on vise notamment à distinguer les passages narratifs des passages dialogués, dans la première rubrique on se centre sur la maîtrise du schéma narratif et de ses composantes, alors que dans la seconde on préconise l'identification de la visée particulière de chaque genre : ainsi, le récit de vie aurait comme visée « informer et intéresser le destinataire»; le témoignage, la visée de « convaincre et restituer des événements »; le fait divers, celle de « rendre compte d'un événement proche » et la biographie celle d' " enrichir les connaissances » (ibid., p. 26).

(3) Pour ceux qui connaissent le système suisse romand, il s'agit des $5^{\mathrm{e}}, 6^{\mathrm{e}}, 7^{\mathrm{e}}$ et $8^{\mathrm{e}}$ degrés Har$\operatorname{moS}$. 
La distinction entre « texte qui argumente » et « texte qui transmet des savoirs » semble tenir moins à la présence (ou non) d'un thème controversé à débattre qu'au caractère interactif (ou non) des constructions textuelles : la première catégorie comprend des genres d'échange écrit comme la lettre d'opinion ou la réponse au courrier des lecteurs, la seconde comprend notamment le texte documentaire et l'article encyclopédique. Quant aux apprentissages à réaliser, dans les deux cas ils portent sur le repérage des organisateurs et des phases du plan de texte, ainsi que sur l'identification de la visée spécifique de chaque genre, mais alors qu'une liste de visées spécifiques est proposée pour «le texte qui argumente » (exprimer une demande, donner son avis, réagir à l'opinion d'autrui, etc.), aucun exemple de telle visée n'est mentionné pour « le texte qui transmet des savoirs ».

La classe des « textes qui règlent des comportements » inclut les genres recette, marche à suivre d'un bricolage, règle du jeu, règlement, etc., et requiert des apprentissages ayant trait à l'identification des actions ou prescriptions évoquées et à leur organisation chronologique.

La classe des « textes qui jouent avec la langue » inclut les genres poème et paroles d'une chanson, et l'un des apprentissages qui y est associé concerne l'identification de la visée de chaque exemplaire de texte de ces deux genres, à savoir raconter, argumenter, décrire, faire rêver, émouvoir, etc.

Il nous paraît possible de dégager de cette brève analyse quatre constats généraux ayant trait au statut accordé aux genres textuels ainsi qu'aux problèmes que pose le classement qui en est proposé.

1. Les approches de Volochinov, du courant francophone d'analyse de discours, aussi bien que celles d'Adam (1990 ; 2011), Bronckart (1997) ou Rastier (2001), mettent en évidence la dimension praxéologique première de la généricité, c'està-dire l'interdépendance entre genres textuels d'une part, sortes d'activités humaines et sphères sociales, professionnelles ou culturelles de leur réalisation d'autre part. Or, comme dans la plupart des documents pédagogiques, cette dimension n'est nullement exploitée dans le regroupement du PER, et fait place à un classement fondé sur des critères qui se donnent pour cognitifs, mais qui en réalité mélangent les trois dimensions qu'avaient identifiées Petitjean ou Schneuwly ( $c f$. 1.1, supra), à savoir le critère procédural (mode d'organisation du contenu : raconter), le critère énonciatif (position à l'égard du cadre communicatif : argumenter vs transmettre) et le critère de fonction psychologique (régler des comportements vs jouer avec la langue).

2 . Il résulte de cette hétérogénéité critérielle que les classes établies ne sont nullement mutuellement exclusives (un texte «qui relate» peut évidemment aussi « transmettre des savoirs »), situation qui entraîne l'identification de «visées » non plus supra-ordonnées aux genres, mais infra-ordonnées : les genres argumentatifs qui, soit expriment un avis, soit réagissent à une opinion, etc. A l'évidence, il y a confusion quant au niveau d'analyse dans lequel faire intervenir les critères de fonction ou de visée.

3. Bien que, pour « le texte qui raconte», soit posée une distinction entre «passages narratifs » et « passages dialogués », le document ne fournit aucune indication générale sur la distinction entre le niveau du " genre» dans son ensemble, et celui de ces " passages » inclus dans les genres, c'est-à-dire de ces structures infra-ordonnées qui ont été conceptualisées soit en termes de « séquences » (Adam), soit en termes de «types de discours » (Bronckart). Or, c'est bien au niveau de ces 
structures, et non du genre dans son ensemble, que sont régies les conditions d'emploi des temps des verbes, des anaphores ou des organisateurs, qui sont les principaux objets dont la maîtrise est visée dans les quatre thématiques du programme Langues - Français.

4. Les étiquettes de genres sont soit issues de la tradition des études littéraires (fable, conte, légende, etc.), soit ont été créées pour l'occasion didactique (marche à suivre d'un bricolage, témoignage d'une expérience vécue, etc.), mais aucune indication n'est fournie sur les conditions et critères permettant de considérer qu'un exemplaire de texte relève bien d'un de ces genres; il n'y a, en d'autres termes, aucune forme de réflexion sur le statut même de cette généricité première.

En dépit des problèmes relevés ci-dessus, il convient de constater que le programme présenté dans ces quatre thématiques ayant trait à la langue satisfait pleinement, en soi, au principe de diversification des genres à enseigner, et que les apprentissages (des régularités d'organisation textuelle) qu'il vise sont absolument pertinents. Les questions qui se posent dès lors sont celle de la possibilité d'introduire en ce domaine des systèmes d'étiquetage et de classification cohérents et enseignables, et en corollaire celle de la pertinence didactique même des démarches d'identification de classes de genres textuels; nous y reviendrons sous 3 , infra.

\subsection{Les genres textuels et leur traitement dans MMF}

Le Livre du maître de la série MMF comporte une Introduction générale, suivie de séquences d'activités renvoyant aux domaines de la littérature, des mathématiques, des sciences, de l'histoire, de la géographie, et de l'éducation à la citoyenneté $^{(4)}$. L'analyse ci-dessous porte d'une part sur deux parties de l'introduction intitulées « Choix didactiques et pédagogiques » et « Fonctionnement de la langue », d'autre part sur une séquence de littérature de $7^{\mathrm{e}}$, ayant pour sous-titre « récit de vie $»$.

Dans le texte d'introduction, le premier choix didactique annoncé consiste à se donner comme objet d'enseignement central le texte, considéré comme « une unité minimale de sens » et défini plus largement dans les termes qui suivent:

Nous proposons de travailler, à l'oral comme à l'écrit, sur ce que nous considérons comme l'unité minimale de sens : LE TEXTE, car « tout texte est pris dans une structure de communication, ce qui n'est pas le cas de la phrase ». Mais il convient de préciser l'objet que nous proposons aux élèves d'explorer, en reprenant l'affirmation de Rastier selon qui, « tout texte relève d'un genre et tout genre d'un dis$\operatorname{cours}^{(5)}$. (MMF, 2010, p. 5)

Ce choix témoigne d'une réticence à l'égard d'un enseignement qui solliciterait de manière trop exclusive les types de textes, dans la mesure où :

(4) La distribution de ces domaines disciplinaires est légèrement différente selon les degrés : par exemple, au $5^{\mathrm{e}}$, il n'y a pas de séquences d'histoire ou de géographie ; celles-ci sont par contre présentes au $6^{\mathrm{e}}$, mais ce degré ne comporte pas de séquence d'éducation à la citoyenneté, etc. Le seul domaine omniprésent est celui de la littérature. S'agissant de 1'Introduction générale, et comme nous l'avons déjà mentionné, elle est commune à tous les degrés et comporte : un Avant-propos adressé aux enseignants du deuxième cycle en Suisse romande, une partie explicitant les choix didactiques et pédagogiques du manuel, une partie consacrée au fonctionnement de la langue (FDL), une partie concernant le système graphique en français, enfin un ensemble de références $F D L$.

(5) Dans les extraits cités de ce manuel, les soulignements en gras sont du texte même. 
[...] un type de texte est une configuration formelle scientifiquement définie plus qu'une réalité sociale [et en conséquence], nous ne voulons pas enfermer les apprenants dans une analyse trop formaliste et abstraite qui finit par ressembler à des exercices formels fonctionnant à vide. (ibid.)

Les objectifs d'apprentissage annoncés témoignent du double souci d'intégration des dimensions de sens et de forme, et de travail sur des productions verbales diversifiées, comme semble l'indiquer le passage ci-dessous :

Notre ambition est de faire travailler conjointement le sens et la forme, donc d'intégrer l'étude de l'énonciation et des genres à l'étude des textes. [...] nous nous donnons comme objectifs de faire travailler les élèves sur des formes diversifiées de parole, qui existent dans les pratiques sociales (école comprise évidemment). (ibid.)

Plus spécifiquement, le travail didactique ayant trait aux textes vise à développer simultanément trois types de compétences définies comme suit : - la compétence textuelle, requérant de l'élève « qu'il repère ou mette en œuvre une suite de phrases organisée et cohérente »; - la compétence discursive, requérant qu'il sache « déceler ou montrer une intention précise, qui s'adresse à un destinataire clairement identifié »; - la compétence sémantico-lexicale, requérant qu'il sache « appréhender un contenu, activer et mettre en lien des connaissances du monde au travers de réseaux de mots $»$.

Quant aux activités relatives au fonctionnement de la langue, elles sont conçues comme occasions de réfléchir à " la manière dont un sujet use de sa langue pour comprendre et se faire comprendre » (p.8), et on relèvera que parmi les « besoins d'apprentissage » en ce domaine est mentionnée « la caractérisation des types de textes » (p. 9).

La séquence Littérature - récit de vie comporte un ensemble d'activités susceptibles de se déployer sur 10 séances de travail dans lesquelles s'entrecroisent des activités dont les objectifs d'apprentissage concernent tous les sous-domaines de la discipline Français. Trois ensembles d'activités ont trait à la problématique du texte : le premier concerne la reconnaissance du genre « récit de vie », qui ne donne lieu à aucun travail de comparaison ou de tri de textes, mais se borne à l'identification du type de contenu référentiel censément spécifique du genre, ainsi qu'à l'identification des champs lexicaux qui seraient « en lien avec les intentions de l'auteur $»$; le second ensemble consiste en l'identification ou la production de dialogues rapportés et de « récits enchâssés », et le dernier ensemble comporte des exercices sur les conditions d'emploi des temps du verbe et des reprises nominales.

Cet examen montre d'abord que la problématique de la généricité a complètement disparu du manuel analysé : celui-ci ne propose aucune approche organisée des genres de textes et de leur possible classement, et les potentielles spécificités des genres sont saisies sous le seul angle thématique, et non sous l'angle de leurs propriétés linguistiques et compositionnelles. Cet examen montre ensuite que le mode d'organisation des activités ne permet nullement, d'une part d'assurer un minimum de représentativité des textes travaillés et donc de satisfaire au principe même de diversification, d'autre part d'assurer que les principales règles d'organisation des textes feront l'objet d'un programme d'apprentissage sous-tendu par le principe de progression didactique.

Le choix de cette série de manuels est ainsi profondément déroutant à deux égards au moins. Tout d'abord, sa compatibilité supposée avec le PER est, du 
moins dans le domaine de l'enseignement des genres, absolument introuvable, dans la mesure où l'on n'y décèle aucune trace de la manière dont la problématique des genres et de leur classement y est traitée ; cela nous semble découler du fait que, dans son ambition d'intégrer les différentes dimensions du travail sur la langue, la démarche de MMF fait quasiment disparaître toute systématique d'organisation des savoirs à enseigner. Ensuite, alors qu'en dépit des difficultés relevées, le PER est le produit d'un sérieux travail de sollicitation et de transposition de références théoriques, la série MMF est truffée d'inexactitudes («le texte est l'unité minimale de sens »? ? ?), de formules creuses au statut d'alibi théorique («tout texte est pris dans une structure de communication, ce qui n'est pas le cas de la phrase »; ou «travailler conjointement le sens et la forme, donc intégrer l'étude de l'énonciation et des genres à l'étude des textes»), de contradictions internes ( u un type de texte est une configuration formelle [...] [et] nous ne voulons pas enfermer les apprenants dans une analyse trop formaliste », en même temps que « la caractérisation des types de textes » est considérée comme un «besoin d'apprentissage ») et de confusions conceptuelles que l'on avait pu croire définitivement bannies des ouvrages didactiques, comme celle subsistant de fait entre les notions d'auteur et de narrateur.

\section{Quelques propositions théoriques et didactiques}

Nous formulerons pour clore un ensemble de réflexions et propositions, qui nous paraissent à même, non pas de couvrir l'étendue de problématiques que soulève le statut des classifications des genres de textes eu égard à l'enseignement de ces derniers, mais de fournir quelques orientations de clarification en ce domaine ; clarification dont le besoin se fait toujours sentir, comme l'ont montré les analyses qui précèdent.

1. Nous soutiendrons d'abord que, en dépit des redoutables questions théoriques et didactiques que cette notion pose, le genre constitue un objet d'enseignement fondamental dans un programme de langue, pour quatre raisons au moins. Premièrement, et comme 1'a montré récemment Bronckart (2013, sous presse), parce que les genres de textes constituent « les cadres organisateurs de la "vraie vie" des signes » de la langue, ces derniers ne se constituant, ne se transformant et ne prenant leurs valeurs significatives que dans la mesure où ils sont mis en œuvre et en circulation dans des textes. Deuxièmement, et comme l'a montré avec clarté Schneuwly, en raison du rôle que les genres jouent dans le développement psychologique humain. Pour cet auteur, le genre peut être considéré comme un « mégaoutil » c'est-à-dire comme un moyen de connaissance, non seulement de la diversité des formes d'expression langagière, mais aussi des activités et des enjeux auxquels les genres sont socialement articulés ou adaptés :

On pourrait $[\ldots]$ considérer le genre comme un « méga-outil», comme une configuration stabilisée de plusieurs sous-systèmes sémiotiques [...] permettant d'agir efficacement dans une classe bien définie de situations de communication. (1994, p. 162).

D'une part le genre [...] doit pouvoir être adapté à un destinataire précis, à un contenu précis, à un but donné dans une situation donnée [...] Mais ce schème d'utilisation fonctionne également dans l'autre sens : une situation ne peut être conçue, connue comme $[\ldots]$ supportant une action langagière d'un certain type que dans la mesure où un genre est disponible. (ibid., p. 161) 
Troisièmement, et comme l'a soutenu Garcia-Debanc (op. cit.), en raison de l'ouverture vers des apprentissages cognitifs plus généraux que permet le travail sur et avec les genres, en particulier la démarche différentielle et comparative impliquée dans le tri de textes. Quatrièmement, en raison du rôle proprement médiateur que le genre textuel nous paraît jouer, cette fois entre paramètres externes de la (situation de) communication et propriétés linguistiques internes aux textes. Comme nous l'avons mentionné, ces dernières sont attestables à des niveaux d'organisation textuelle infra-ordonnés, conceptualisés en termes de «types de discours » ou de « séquences », qui n'entretiennent pas de lien direct avec les paramètres externes de la communication. Dès lors, et c'est précisément en cela que consiste l'une de ses spécificités majeures, c'est le genre qui assure l'interface entre extra-linguistique et linguistique dans la mise en forme textuelle.

2. En lien avec ce qui précède, nous soulignerons l'utilité d'un travail articulé aux principes de diversification et de représentativité, qui consisterait à comparer et trier des exemplaires de genres en commentant librement des classements possibles ; mais, si l'attitude de détachement ( $c f .1 .2$, supra) prônée par certains didacticiens est en ce domaine d'une indiscutable pertinence, ce travail nous paraît en même temps devoir tenir compte premièrement de critères praxéologiques, à savoir les rapports des genres aux activités sociales, professionnelles et culturelles générales, et leurs rapports aux média et supports qui véhiculent les textes. Ces critères nous paraissent devoir être soigneusement distingués, à la fois au plan théorique et didactique, d'une part de ceux qui concernent les paramètres de la situation de production des textes - cette dernière n'étant nullement assimilable ou superposable à l'activité sociale à laquelle un genre est articulé, ni au média de diffusion de celui-ci - d'autre part de ceux qui portent sur les modes d'organisation interne des textes. Relevons encore que, au plan méthodologique, cette distinction est de fait impliquée par la différenciation des niveaux d'analyse qu' avait conceptualisée Volochinov ( $c f .1 .1$, supra); et que, au plan terminologique, elle impliquerait d'éviter les appellations, pourtant si courantes, de " genres narratifs », « genres argumentatifs », etc., qui projettent sur le niveau de la généricité des propriétés relevant de modes linguistiques spécifiques, et/ou de niveaux d'organisation textuelle interne.

3. Au plan proprement didactique, la distinction évoquée ci-dessus autorise, voire requiert, une autre distinction encore, entre d'une part les activités portant sur les genres textuels à proprement parler, ayant comme finalité de sensibiliser les élèves à la diversité textuelle et générique ainsi qu'à l'articulation entre genres textuels et sphères d'activité humaine, et d'autre part les activités visant la maîtrise de l'organisation textuelle interne, qui porteraient sur les structures infra-ordonnées comme les séquences ou les types de discours, ou encore sur d'autres mécanismes de textualisation. C'est seulement dans un deuxième temps, et suite à ces activités différenciées, que devrait être posée la question du rapport entre généricité textuelle et propriétés linguistiques dominantes et/ou récurrentes, ou encore celle de l'influence d'un certain genre sur le mode de manifestation de certains mécanismes linguistiques. Cette question déboucherait ainsi effectivement, entre autres, sur le constat de la prédominance d'un certain type de discours, ou d'une certaine catégorie de séquences, à l'intérieur d'un (genre de) texte ; phénomène qui re-pose, nous semble-t-il, la question du statut des appellations « genres narratifs », « argumentatifs », etc., en même temps qu'il fournit une explication potentielle de la difficulté de s'en défaire, comme il ressort de la citation ci-dessous : 
De nombreux genres discursifs fixent, avec plus ou moins de latitude, le type de forme première dominante. Ainsi les genres du conte et de la fable sont-ils narratifs tandis que le genre épistolaire (avec ses sous-genres : courrier personnel, administratif, courrier des lecteurs dans la presse, etc.), l'interview, le théâtre doivent être considérés comme des genres conversationnels et le guide touristique comme un genre à dominante descriptive. (Adam, 2011, p. 28)

Néanmoins, respecter ces temps didactiques distincts permettrait de traiter ces désignations sur des bases notablement différentes, en ce qu'elles contribuent à clarifier les niveaux auxquels il est pertinent de les associer, à saisir éventuellement les raisons de ce " deuxième degré » possible d'appellation, et surtout à dénaturaliser (ou éviter de naturaliser) le statut linguistique de la généricité — ce qui ne rend pas pour autant cette dernière moins langagière ou moins sémiotique !

4. Enfin, au plan de la formation des enseignants, et s'agissant en particulier de la formation continue, l'explicitation conjointe de la pertinence intrinsèque des divers processus classificatoires des genres et des difficultés inhérentes à ce classement pourrait constituer un véritable objet de formation; la visée de ce type d'analyse n'étant nullement la maîtrise en termes de contenu de ces phénomènes, mais l'acquisition d'outils pour la compréhension des raisons qui conduisent potentiellement à des contradictions dans les documents didactiques, et surtout la (réelle) sensibilisation au statut même de la généricité, telle qu'elle est problématisée, par exemple, par Adam \& Heidmann :

L'étiquette genre - les noms de genres « conte de fées », " Märchen », « tragédie », " fait divers ", etc. — ont tendance à réduire un énoncé à une catégorie de textes. La généricité est, en revanche, la mise en relation d'un texte avec des catégories génériques ouvertes. Cette mise en relation repose sur la production et/ou la reconnaissance d'effets de généricité, inséparables de l'effet de textualité. [...] La généricité est une nécessité socio-cognitive qui relie tout texte à l'interdiscours d'une formation sociale. Un texte n'appartient pas, en soi, à un genre, mais il est mis, à la production comme à la réception-interprétation, en relation à un ou plusieurs genres.

[...] Il s'agit d'aborder le problème du genre moins comme l'examen des caractéristiques d'une catégorie de textes que comme la prise en compte et la mise en évidence d'un processus dynamique de travail sur les orientations génériques des énoncés. Ce travail s'effectue sur les trois plans de la production d'un texte, de sa réception-interprétation et sur le plan intermédiaire très important de son édition. (Adam \& Heidmann, 2004, p. 62-63)

Une formation des enseignants à la généricité qui serait, en définitive, l'occasion de comprendre non seulement les sources de leurs possibles déroutes, mais, plus important encore, la socio-historicité et la dynamicité qui caractérisent aussi bien les genres textuels que les démarches d'étiquetage, de connaissance et d'enseignement qui leur sont appliquées. 


\section{Bibliographie}

ADAM, J.-M. (1990). Eléments de linguistique textuelle. Liège : Mardaga.

- (1991). « Cadre théorique d'une typologie séquentielle ». Etudes de linguistique appliquée, 83, 7-18.

- (2011). Les textes : types et prototypes. Paris : Armand Colin [ $1^{\text {re }}$ édition : 1992].

ADAM, J.-M. \& HEIDMANN, U. (2004). «Des genres à la généricité. L'exemple des contes (Perrault et les Grimm) ». Langages, 153, 62-72.

BAIN, D. (1987). «D'une typologie à une didactique du texte». Enjeux, 11, 49-68.

Bain, D., Davaud, C., Bronckart, J.-P., PAsquier, A. \& Schneuwly, B. (1982). Classification de textes pour la rédaction et l'analyse. Genève : Cahiers de la Section des Sciences de l'Education, 28, 74 p.

BARThes, R. (1984). Le bruissement de la langue. Paris : Seuil.

BENVENISTE, E. (1966). «Les relations de temps dans le verbe français ». In Problèmes de linguistique générale, $t$. I, (237-250). Paris : Gallimard.

Brassart, D.G., CAUterman, M.-M., Darras, F., DelCAMBre, I. \& GraCZYK, B. (1986). «Les trains, $1^{\text {ère }}$ classe, $2^{\text {ème }}$ classe..., de la $6^{\mathrm{e}}$ à la $4^{\mathrm{e}}$. Typologie des textes et des discours ». Repères, 69, 87-105.

BRONCKART, J.-P. (1991). Perspectives et limites d'une diversification de l'enseignement du français. Etudes de linguistique appliquée, 83, 63-74.

- (1997). Activité langagière, textes et discours. Pour un interactionisme socio-discursif. Paris : Delachaux et Niestlé.

- (2013, sous presse). «Les genres de textes, cadres organisateurs de la «vraie vie » des signes ». In M. Monte \& B.G. Philippe (Ed.) Genres et textes : déterminations, évolutions, confrontations. Hommage à J.-M. Adam. Lyon : PUL.

Bronckart, J.-P., Bain, D., Schneuwly, B., Davaud, C. \& Pasquier, A. (1985). Le fonctionnement des discours. Un modèle psychologique et une méthode d'analyse. Paris : Delachaux et Niestlé.

BronckART, J.-P. \& BotA, C. (2011). Bakhtine démasqué. Histoire d'un menteur, d'une escroquerie et d'un délire collectif. Genève : Droz.

CHISS, J.-L. (1987). « Malaise dans la classification ». Langue française, 74, 10-28 [repris dans Chiss, J.-L. (2012). L'écrit, la lecture et l'écriture. Théories et didactiques. L'Harmattan, p. 23-46.]

Coste, D. (1991). « Genres de textes et modes discursifs dans l'enseignement/apprentissage des langues ». Etudes de linguistique appliquée, 83, 75-88.

Dolz, J. \& SCHNeUWly, B. (1998). Pour un enseignement de l'oral. Initiation aux genres formels à l'école. Paris : ESF.

ETUDES DE LINGUISTIQUE APPLIQUÉE, 83 (1991). Textes, discours, types et genres . (Ed. : J.-P. Bronckart, D. Coste \& E. Roulet).

FILliolet, J. (1987). «La typologie des discours, mythe ou réalité pédagogique?», Langue française, 74, 97-107.

GARCIA-DEBANC, C. (1989). «Le tri de textes : modes d'emploi».Pratiques, 62, 351.

KINTSCH, W. \& VAN DIJK, T. (1978). « Toward a model of text comprehension and production ». Psychological Review, 85, 163-182. 
LANGUE FRANÇAISE, 74 (1987). La typologie des discours. (Ed. : J.-L. Chiss \& J. Filliolet).

Maingueneau, D. (1984). Genèses du discours. Bruxelles : Mardaga.

MAS, M. \& TURCO, G. (1991). " Des typologies de textes à l'élaboration d'outils pour la formation des maitres et d'outils pour la classe ». Etudes de linguistique appliquée, 83, 89-99.

Moreau, M.-L. \& Mouvet, B. (1990). « Je vous fais une lettre : différencier la didactique de l'écriture épistolaire ». In B. Schneuwly (Ed.), Diversifier l'enseignement du français écrit (p. 110-114). Paris : Delachaux et Niestlé.

Petitjean, A. (1989). «Les typologies textuelles ». Pratiques, 62, 86-125.

PRATIQUES, 62 (1989). Classer les textes. (Ed. : A. Petitjean).

PRIVAT, J.-M. \& VINSON, M.-C. (1988). « Tableaux de genres : travailler les critères de genres en lecture-écriture ». Pratiques, 59, 3-17.

RAstier, F. (2001). Arts et sciences du texte. Paris : PUF.

SCHMidT, S.J. (1976). Texttheorie. München : Wilhelm Fink Verlag.

SCHNEUWLY, B. (1988). «Quelle typologie pour l'enseignement? Une typologie des typologies ». In J.-L. Chiss et al. (Ed.), Apprendre/enseigner à produire des textes écrits (p. 53-64). Bruxelles : Duculot.

- (1991). «Diversification et progression en DFLM : 1'apport des typologies ». Etudes de linguistique appliquée, 83, 131-141.

- (1994). "Genres et types de discours : considérations psychologiques et ontogénétiques ». In Y. Reuter (Ed.), Les interactions lecture-écriture (p. 155-174). Bern : Lang.

SCHNEUWLY, B. \& DOLZ, J. (1997). « Les genres scolaires. Des pratiques langagières aux objets d'enseignement $»$. Repères, 15, 27-41.

SimONin-GRUMBACH, J. (1975). «Pour une typologie des discours ». In J. Kristeva, J.-C. Milner \& N. Ruwet (Ed.), Langue, discours, société. Pour Emile Benveniste (p. 85-121). Paris : Seuil.

TODOROV, T. (1978). Les genres du discours. Paris : Seuil.

VAN DiJK, T. (1972). Some aspects of text-grammars. The Hague : Mouton.

Volochinov, V. (2010). Marxisme et philosophie du langage. Limoges : LambertLucas [Edition originale : 1929].

VOLOCHINOV, V.N. (1981a). « Le discours dans la vie et le discours en poésie ». In

T. Todorov (Ed.), Mikhaïl Bakhtine le principe dialogique (p. 181-215). Paris : Seuil [Edition originale : 1926].

WEINRICH, H. (1973). Le temps. Paris : Seuil.

WERLICH, E. (1975). Typologie der texte. Heidelberg: Quelle \& Meyer.

\section{Documents analysés}

Mon Manuel de français. Livre du maître $+C D$ audio (2010). Paris : Retz.

Plan d'Etudes Romand. (2010). Documents disponibles sur le site de la CIIP. http ://www.plandetudes.ch/documents/10136/19192/cycle 2 webCIIP.pdf 